\title{
4- and 5-level anterior fusions of the cervical spine: "natura non-facit saltus"
}

\author{
João Luiz Pinheiro Franco
}

Received: 27 March 2008/Accepted: 18 May 2008/Published online: 18 June 2008

(C) Springer-Verlag 2008

I read with interest the article from Drs. Koller H, Hempfing A, Ferraris L, Maier O, Hitzl W, Metz-Stavenhagen P: "4- and 5-level anterior fusions of the cervical spine: review of literature and clinical results". Eur Spine J. 2007 December; 16(12):2055-71.

The authors performed a case review of consecutive 26-instrumented 4- and 5-level anterior cervical fusions trying to address some of the puzzling questions in multilevel anterior cervical surgeries. In fact they did a remarkable review on the biomechanics and on the sagittal balance $^{1}$ after multilevel surgery. Important concepts were presented and discussed. A very important work was done considering the relative paucity of articles concerning 4- and 5-level anterior cervical disk fusions.

However, some important issues were not argued in detail. Dr. Koller et al. stated that "indications for multilevels surgery included symptomatic degenerative cervical instability and cervical stenosis with either radiculopathy, myelopathy or axial neck pain as well as pseudoarthrosis, implant failure or adjacent disc disease following previous surgeries...". The authors here failed to give a better description of exactly what do they consider to indicate multilevel decompression and fusion. How to define the levels to be decompressed? Obviously looking at the MRI provides a good appreciation of which levels need to be decompressed. But, let us remind a phrase that makes echoes in our brains when considering lumbar DDD, "we

A reply on this paper is available at doi: 10.1007/s00586-008-0698-6.

J. L. P. Franco $(\square)$

Neurosurgery Department, Samaritano Hospital,

São Paulo, Brazil

e-mail: jlpfranco@uol.com.br do not operate images, we consider clinics and images". So, how to proceed? Look at the MRI and program surgery? In this case, we do not need the patient to come to our offices... Patients with a predominant myelopathic presentation generally provide an easier decision-making as the symptoms come from the axial compromise and therefore, all the compressed levels must be decompressed. In patients where radiculopathy prevails, the answer is more difficult. What about the clinical presentation of these "radiculopathic" patients: do they have a clear cervical nerve pain or maybe a kind of a truncated, diffuse ache? Did the authors perform ENMG tests? How to define which levels to approach? Sometimes we do want to answer complex answers, but we forget the basis, the initial steps. As the latin proverb says: "Natura non-facit saltus" (Nature/life is not made of jumps). This kind of information would be extremely helpful for young surgeons and quite interesting as scientific information exchange between experienced ones.

Pinheiro-Franco et al. (personal communication) studied prospectively a cohort of 23 consecutive patients with multilevel cervical anterior compression. He infrequently added $\mathrm{C} 2-\mathrm{C} 3$ to the fusion once the spinal canal diameter is larger at this level. When myelopathy was the major presenting symptom, this author defined the longitudinal extent of decompression simply through MRI analysis. Notwithstanding, when there were no signs of myelopathy, the answer was not so easy. Debate exists concerning if axial pain per se plus MRI multilevel anterior cervical

\footnotetext{
1 The nomenclature "sagittal balance" must be preferred than the used "geometry", as Geometry (Greek $\gamma \varepsilon \omega \mu \varepsilon \tau \rho i \alpha$; geo $=$ earth, metria = measure) is a part of mathematics concerned with questions of size, shape and relative position of figures and with properties of space. (http://en.wikipedia.org/wiki/Geometry)
} 
compression would be a good indication for all stenotic levels decompression.

Furthermore, the authors did not comment on important technical details as which kind of retractors do they use? For multilevel pathology, we can imagine that an extensive longitudinal skin incision was performed for all cases. So, how about the retractors for these 4- and 5-level anterior cervical fusions: manual or statics? One of the possible morbidities from anterior cervical disk fusion (ACDF) is temporary dysphagia, possibly linked to the time spent on esophagus retracting. The authors did not mention their technique and provided a $15.4 \%$ of postoperative temporary dysphagia. How do the authors measured the degree of dysphagia? Are the minor complaints being counted? A recent study from Edwards, John Heller, Daniel Riew et al. concluded that postoperative dysphagia is underreported in $80 \%$ of the cases by the surgeon $[1,2]$. Other authors published recently, results from a prospective investigation, the incidence of postoperative dysphagia being 50.2, $32.2,17.8$ and $12.5 \%$ at $1,2,6$ and 12 months, respectively [3]. Therefore, despite large incisions for 4- and 5-level surgery, with esophagus traction, $15.4 \%$ of postoperative temporary dysphagia may be considered an acceptable value and would deserve a technical explanation for the readers.

Finally technical details regarding approach and plate and screws positioning for C7-T1 levels were not discussed. It would be interesting to have the authors' experience exposed.

Again, I congratulate the authors for their whole work on this demanding scarce published 4- and 5-level ACDF.

\section{References}

1. Edwards CC 2nd, Karpitskaya Y, Cha C, Heller JG, Lauryssen C, Yoon ST, Riew KD (2004) Accurate identification of adverse outcomes after cervical spine surgery. J Bone Joint Surg Am 86$\mathrm{A}(2): 251-256$

2. Fountas KN (2007) Re: Papavero L, Heese O, Klotz-Regener V et al (2007) The impact of esophagus retraction on early dysphagia after anterior cervical surgery. Spine 32:1089-1093. Spine 32(26):3090

3. Bazaz R, Lee M, Yoo J (2002) Incidence of dysphagia after anterior cervical spine surgery: a prospective study. Spine 27(22):2453-2458 